\title{
Rice Germin-Like Proteins: Allelic Diversity and Relationships to Early Stress Responses
}

\author{
Rebecca M. Davidson • Patricia M. Manosalva • \\ Jacob Snelling • Myron Bruce $\cdot$ Hei Leung • \\ Jan E. Leach
}

Received: 25 September 2009 / Accepted: 26 January 2010/Published online: 25 February 2010

(C) Springer Science+Business Media, LLC 2010

\begin{abstract}
Germin-like protein (GLP) markers were associated with quantitative trait loci (QTL) for resistance to the rice blast pathogen, Magnaporthe oryzae in multiple rice (Oryza sativa) mapping populations. Twelve paralogous OsGLP gene family members are located within the physical QTL region on chromosome 8, and gene silencing studies suggest that they contribute collectively to the resistance phenotype. We compared sequence and expression profiles of $O S G L P$ alleles in two resistant and two susceptible parental rice lines to find functional polymorphisms that correlated with the resistant phenotype. Based on coding and promoter sequences, the genes belong to two germin subfamily groups (GER3 and GER4). OsGLP members from both subfamilies were constitutively expressed and developmentally regulated in all cultivars. Transient induction above constitutive levels was observed for some $O S G L P$ s, especially GER4 subfamily members, at early time points after M. oryzae infection and mechanical wounding. Varying $5^{\prime}$ regulatory regions and differential expression of some family members between resistant and
\end{abstract}

Electronic supplementary material The online version of this article (doi:10.1007/s12284-010-9038-7) contains supplementary material, which is available to authorized users.

R. M. Davidson · P. M. Manosalva · J. Snelling • M. Bruce •

J. E. Leach $(\bowtie)$

Department of Bioagricultural Sciences and Pest Management and Program in Plant Molecular Biology,

Colorado State University,

C203 Plant Sciences Bldg.,

Fort Collins, CO 80523-1177, USA

e-mail: Jan.Leach@colostate.edu

H. Leung

Plant Breeding, Genetics, and Biotechnology,

International Rice Research Institute,

Los Baños, Philippines susceptible cultivars corresponded with differential hydrogen peroxide $\left(\mathrm{H}_{2} \mathrm{O}_{2}\right)$ accumulation after the same stimuli. OsGLP of both GER subfamilies localized to the plant cell wall. The protein location and early gene induction suggest that $O s G L P s$ protect rice leaves at early stages of infection before fungal penetration and subsequent ingress. Our data suggest that regulation of $O S G L P$ genes defines resistant versus susceptible phenotypes.

Keywords Rice $\cdot$ Germin-like proteins $\cdot$ Magnaporthe oryzae $\cdot$ Quantitative trait loci $\cdot$ Disease resistance

\section{Introduction}

Rice blast, caused by Magnaporthe oryzae, is one of the most destructive rice (Oryza sativa) diseases (Willocquet et al. 2004). Genetic resistance controlled by single genes has been widely used to control rice blast, but the resistance is often short-lived due to changes in the fungal population structure (Ballini et al. 2008). Disease resistance governed by quantitative trait loci (QTL), or many genes with small effects, however, is predicted to be durable partially because it exhibits no specificity to a given pathogen group or race and, in some cases, confers resistance to multiple types of pathogens (Leung 2008; Poland et al. 2009). There is good evidence that QTL might control broad-spectrum genetic resistance in some rice populations. A germin-like protein (GLP) gene marker that was associated with a QTL on rice chromosome (chr) 8 conferred resistance against rice blast in multiple rice mapping populations across diverse environments (Ramalingam et al. 2003; Liu et al. 2004; Wu et al. 2004). Twelve paralogous OsGLP genes were identified within the physical QTL region (Manosalva et al. 2009). Members of the $O S G L P$ family were shown to contribute 
collectively to disease resistance because silencing of several genes confers susceptibility to two distinct fungal pathogens, M. oryzae and Rhizoctonia solani, the sheath blight pathogen (Manosalva et al. 2009). The susceptible phenotypes of the $O s G L P$-silenced mutants confirm roles for some gene family members, particularly those in the GER4 subfamily, in broad-spectrum disease resistance (Manosalva et al. 2009).

The rice chr 8 OsGLPs belong to two subfamilies of germins, the GER3 and GER4 (Davidson et al. 2009; Manosalva et al. 2009). These subfamilies were also described in barley (Hordeum vulgare), wheat (Triticum aestivum), grapevine (Vitus viniferous), and Arabidopsis thaliana (Schweizer et al. 1999; Membre et al. 2000; Zimmermann et al. 2006; Godfrey et al. 2007). GLP genes in these subfamilies are developmentally regulated and constitutively expressed in multiple tissue types including leaves, roots, and flowers (Dunwell et al. 2008; Davidson et al. 2009). Many of these genes are induced by fungal and bacterial infections, and some produce proteins with superoxide dismutase (SOD) activity (Schweizer et al. 1999; Christensen et al. 2004; Godfrey et al. 2007; Davidson et al. 2009). GLP gene induction was observed in rice after infection with M. oryzae (Davidson et al. 2009; Manosalva et al. 2009), in barley and wheat leaves after infection with the powdery mildew pathogen (Wei et al. 1998; Christensen et al. 2004; Zimmermann et al. 2006), in barley spikelets after infection with Fusarium graminearum (Federico et al. 2006), and in grapevine leaves and fruit after infection with Erysiphe necator (Godfrey et al. 2007). Transcripts of wheat and barley GER3 and GER4 genes accumulated predominantly in epidermal cells compared to mesophyll cells as early as $6 \mathrm{~h}$ post-inoculation (hpi; Wei et al. 1998; Schweizer et al. 1999; Zimmermann et al. 2006). Interestingly, HvGER4 members were also induced by exogenous application of hydrogen peroxide $\left(\mathrm{H}_{2} \mathrm{O}_{2}\right)$ and the non-host soybean fungus, Phakopspora pachyrhizi (Zimmermann et al. 2006). Consistent with gene silencing results in rice (Manosalva et al. 2009), transient silencing of barley HvGER4d resulted in hypersusceptibility to Blumeria graminis, although silencing of HvGER3a did not (Zimmermann et al. 2006). Genes in both subfamilies show induction by pathogen infection; however, gene silencing of only GER4 subfamily members results in increased susceptibility.

The proposed mechanism by which GLPs provide broadspectrum resistance relates to their SOD activity and localization in plant cell walls (Wei et al. 1998; Membre et al. 2000; Christensen et al. 2004). GLP enzymes with SOD activity convert the superoxide anion into $\mathrm{H}_{2} \mathrm{O}_{2}$ (Woo et al. 2000). Hydrogen peroxide is a player in early plant defense responses and has potential roles in cell wall reinforcement and papillae formation (Wojtaszek 1997; Schweizer et al. 1999), as a signaling molecule for downstream defense responses (Grant et al. 2000; Gechev et al. 2006), and as a toxic defense compound against invading microbes (Shetty et al. 2008). Cell wall localization of GLPs was documented in barley and wheat (Wei et al. 1998; Schweizer et al. 1999), but has not been studied in rice.

We have shown that OsGLPs in the GER4 subfamily function in non-specific resistance to two fungal pathogens (Manosalva et al. 2009). The molecular basis for allelic differences in the involvement of specific $O s G L P \mathrm{~s}$ in QTLbased resistance, however, is still unknown. The goals of this study were to understand the differences among cultivarspecific $O s G L P$ alleles and to functionally link sequence polymorphisms observed in QTL mapping to differential defense responses. Differences between alleles underlying QTL can vary from single nucleotide substitutions to large insertions or deletions between parental cultivars (Paran and Zamir 2003). Some differences may affect coding sequences, while others may occur in upstream regulatory regions and influence gene expression. We predicted defense-related $O S G L P$ family members by comparing the putative promoter regions of $O s G L P$ s from the two reference rice genomes, cv. Nipponbare (ssp. japonica; Yuan et al. 2005) and cv. 93-11 (ssp. indica; Zhao et al. 2004). Then, OsGLP alleles of two rice blast resistant cultivars containing the effective QTL on chr 8 (both ssp. indica) and two susceptible rice cultivars without the chr 8 QTL (both ssp. japonica) were studied for coding sequence polymorphisms and differential gene expression both developmentally and after biotic and abiotic stresses. Furthermore, we confirm the subcellular localization of GER3 and GER4 subfamily members and study $\mathrm{H}_{2} \mathrm{O}_{2}$ peroxide accumulation in QTL-based resistant and susceptible interactions with $M$. oryzae. This information can guide breeding efforts to accumulate desirable alleles from resistant donors into locally adapted cultivars.

\section{Results}

A germin-like protein gene family is located within the chr 8 QTL region

Twelve germin-like protein genes were predicted in a $2.8-\mathrm{Mb}$ region within the proposed rice blast QTL region on rice $\mathrm{chr}$ 8 (Fig. 1). Eleven family members are clustered in a short $80-\mathrm{kb}$ section from approximately 5.18 to $5.26 \mathrm{Mb}$, and the 12th is located downstream at approximately $7.99 \mathrm{Mb}$. Six OsGLP were sequenced and described previously (Table 1). Alignments of gene and protein sequences from the Nipponbare reference genome (MSU Pseudomolecules, Build 4.0; Yuan et al. 2005) indicate that the chr 8 OsGLP are highly similar to each other (data not shown). Pairwise comparisons of genomic sequences range in identity from $47 \%$ to $88 \%$, and corresponding comparisons of inferred amino acid sequences range from $70 \%$ to $98 \%$. The gene 


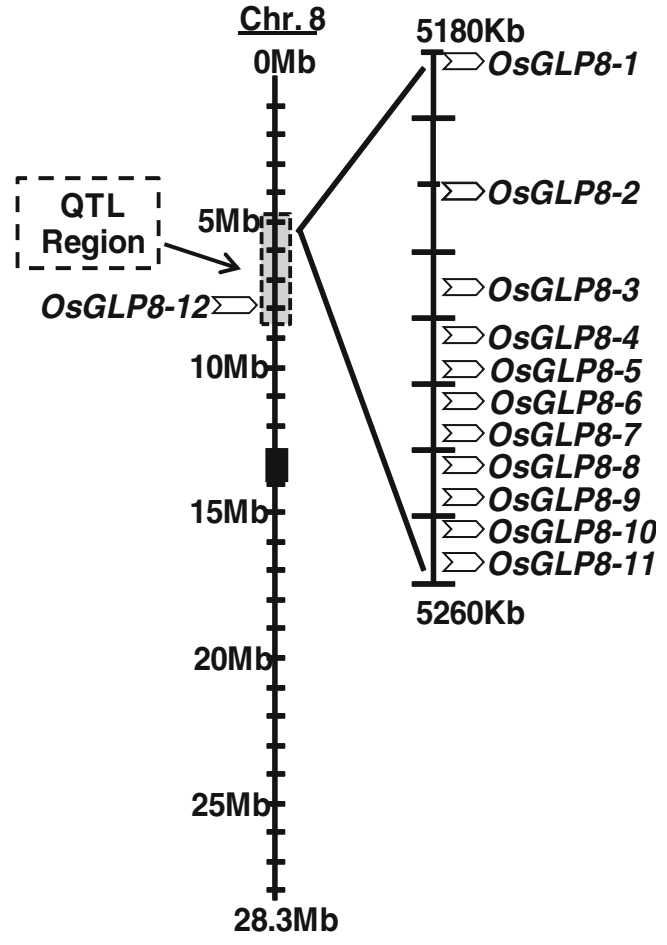

Fig. 1 Distribution of $O s G L P$ family members within the proposed rice blast disease resistance QTL region (shaded box) on chromosome 8 of $O$. sativa. Gene predictions were based on MSU Rice Pseudomolecules, Build 4.0 (Yuan et al. 2005). The chromosome and inset are represented as $1-\mathrm{Mb}$ and $10-\mathrm{kb}$ increments, respectively. The centromeric region is shown as a black rectangle, and relative positions of the 12 OsGLP genes are shown as white arrows.

family members are predicted to be transcribed from the positive strand with the exception of OsGLP8-12. All putative proteins have predicted $\mathrm{N}$-terminal signal sequences of 22-25 amino acids and are predicted to be extracellular.

Phylogenetic reconstruction of $O s G L P$ alleles among five rice cultivars including those from the reference cv.
Nipponbare shows that the chr 8 OsGLP cluster contains genes in two germin subfamily groups, GER3 and GER4 (Electronic supplementary materials (ESM) Fig. 1; Davidson et al. 2009; Manosalva et al. 2009). The GER3 subfamily includes OsGLP8-1, 8-2, 8-3, and 8-12. The GER4 subfamily includes seven members, OsGLP8-5 to 8-11; these are tandemly arranged as a cluster within $30 \mathrm{~kb}$ (Fig. 1) and have pairwise amino acid identities in the range of $90-98 \%$.

Motifs in putative $5^{\prime}$ regulatory regions are shared among $O S G L P$ subfamily members

Bioinformatic comparisons of putative $O S G L P$ promoter sequences from the two rice reference genomes, cv. Nipponbare (ssp. japonica) and cv. 93-11 (ssp. indica) revealed an 858-bp insertion sequence in the indica version of OsGLP8-6 (Fig. 2). The insertion fragment is located $590 \mathrm{bp}$ upstream from the initiation codon (Fig. 2a). The two resistant indica cultivars, IR64 and SHZ-2, contained the insertion, while the susceptible cultivars Azucena and LTH, like the japonica Nipponbare, did not (Fig. 2b).

Shared motif analysis was performed using the 12 putative $O S G L P$ promoter sequences from the Nipponbare reference genome sequence. The insertion sequence in the indica version of the putative promoter of OsGLP8-6 was included in the training set $(N=13)$ to look for additional ciselements. The 15 most statistically overrepresented motifs among the 13 sequences were identified by the MEME algorithm. Each of the 15 motifs was identified in many OsGLP members (ESM Table 1). Motif sequences were analyzed for the presence of known plant cis-elements in the PLACE database (496 entries), and totals were summarized for each putative regulatory sequence (Table 2). With the exception of the TATA box, only those with multiple copies were counted. Sequence degeneracy was observed across

Table 1 Previously Described $O s G L P$ Genes on Rice Chromosome 8

\begin{tabular}{lllll}
\hline Previous name & GenBank acc. no. ${ }^{\mathrm{a}}$ & Locus ID $^{\mathrm{b}}$ & Gene description & New gene name \\
\hline$G E R 3^{\mathrm{c}}$ & AF032973 & LOC_Os08g08960 & cDNA from panicle at ripening & OsGLP8-2 \\
$G L P 16^{\mathrm{e}}$ & $\mathrm{AF042489}$ & LOC_Os08g08960 & cDNA from immature seed & OsGLP8-2 \\
$G E R 2^{\mathrm{c}}$ & AF032972 & LOC_Os08g08970 & cDNA from etiolated shoot & OsGLP8-3 \\
$G E R 1^{\mathrm{c}}$ & AF032971 & LOC_Os08g08980 & cDNA from etiolated shoot & OsGLP8-4 \\
$G E R 6^{\mathrm{c}}$ & AF032976 & LOC_Os08g09010 & cDNA from green shoot & OsGLP8-7 \\
$R G L P 2^{\mathrm{d}}$ & DQ414400 & LOC_Os08g09060 & promoter region from indica & OsGLP8-10 \\
$R G L P 2^{\mathrm{e}}$ & AF141879 & LOC_Os08g09060 & cDNA from rice root & OsGLP8-10 \\
$R G L P 1^{\mathrm{e}}$ & AF141880 & LOC_Os08g09080 & cDNA from rice root & OsGLP8-11 \\
\hline
\end{tabular}

${ }^{\mathrm{a}}$ GenBank gene sequence database (http://www.ncbi.nlm.nih.gov)

${ }^{\mathrm{b}}$ MSU Rice Genome Annotation Database (http://rice.plantbiology.msu.edu/)

${ }^{\mathrm{c}}$ Membre and Bernier (1998)

${ }^{\mathrm{d}}$ Mahmood et al. (2007)

${ }^{\mathrm{e}}$ Unpublished 
a

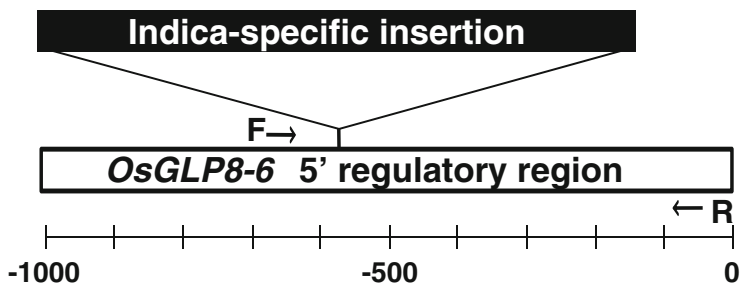

b

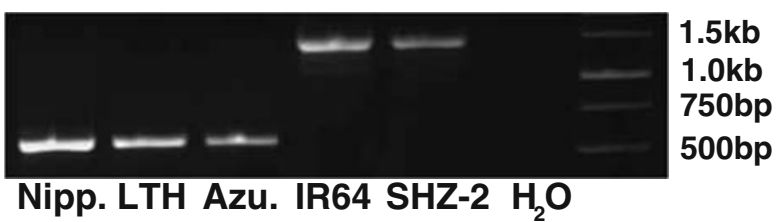

Fig. 2 Allelic alternatives of the $5^{\prime}$ regulatory region in the OsGLP8-6 gene. a An 858-bp insertion was identified in the putative 5' regulatory region of $O S G L P 8-6$ of the 93-11 (ssp. indica) reference genome and was not found in the Nipponbare (ssp. japonica) reference genome. Primers designed upstream of the initiation codon amplified the insertion. b Predicted size products (626 bp) were amplified from the japonica cultivars, Nipponbare, LTH and Azucena and larger sized fragments $(1,478 \mathrm{bp})$ were observed from the indica cultivars, IR64 and SHZ-2.

motif sequence alignments especially in W-BOX and TATA box motifs (ESM Table 1).These are not the only putative cis-elements in the $5^{\prime}$ regulatory regions of OsGLP genes, but rather, they represent motifs that are present in multiple copies and are shared among family members.

Known plant-associated cis-elements were identified within overrepresented motifs (Table 2). TATA box sequences (TATATAA; Grace et al. 2004) which are present just upstream of initiation codons were identified between 77 and $152 \mathrm{bp}$ upstream from the ATG for the 12 OsGLP genes, but not in the OsGLP8-6 insertion sequence. The W-BOX motif [(T)TGAC(Y)], a cis-element associated with rapid induction by wounding or fungal elicitors (Eulgem et al. 1999;
Nishiuchi et al. 2004), was present in multiple copies in all of the putative $5^{\prime}$ regulatory regions and the OsGLP8-6 insertion sequence. More W-BOX elements were discovered in GER4 family members compared to GER3. A pollenspecific cis-element (GTGA; Rogers et al. 2001) and an endosperm-specific element (AAAG; Yanagisawa and Schmidt 1999) were observed in all but two OsGLP regulatory regions, and higher copy numbers were also present in GER4 compared with GER3.

OsGLP alleles show sequence diversity among rice cultivars

Gene sequences of all 12 OsGLP alleles from two resistant cultivars with the chr 8 QTL and two susceptible cultivars without the QTL were analyzed for sequence variation. Single nucleotide polymorphisms (SNPs) were present in one or more cultivars for all genes (Table 3). SNPs predicted to result in non-synonymous codon changes were observed in one or more cultivars for all genes except OsGLP8-6 (Table 3). There were no nucleotide and/or codon substitutions predicted to change protein function that correlated with resistant and susceptible phenotypes. Two OsGLP alleles from the most resistant cultivar, SHZ-2, contained SNPs conferring premature stops. A 1-bp deletion in OsGLP8-1 caused a frameshift mutation, and a nucleotide substitution in OSGLP8-11 resulted in a premature stop codon.

Expression of $O S G L P$ genes is developmentally regulated

To examine the constitutive and developmental expression of $O s G L P$ genes, expression was monitored using gene-specific primers for all 12 genes in three positions of non-stressed leaves in three cultivars: Azucena, IR64, and SHZ-2 (Table 4). Transcripts for OsGLP8-1, 8-2, and 8-10 were

Table 2 Numbers of Plant cis-Elements in Statistically Overrepresented Motifs Among OsGLP Gene Family 5' Regulatory Regions

\begin{tabular}{|c|c|c|c|c|c|c|c|c|c|c|c|c|c|c|}
\hline \multirow[t]{2}{*}{ cis-Element } & \multicolumn{14}{|c|}{$O s G L P 8$ promoters } \\
\hline & 1 & 2 & 3 & 4 & 5 & 6 & 7 & 8 & 9 & 10 & 11 & 12 & 6 insert. $^{\mathrm{a}}$ & Functional association \\
\hline AAAG & 1 & 2 & 0 & 1 & 2 & 3 & 3 & 3 & 3 & 3 & 0 & 1 & 1 & Endosperm-specific Yanagisawa and Schmidt (1999) \\
\hline GTGA & 1 & 1 & 1 & 0 & 1 & 2 & 3 & 3 & 3 & 2 & 1 & 0 & 1 & Pollen-specific (Rogers et al. 2001) \\
\hline TATATAA & 1 & 1 & 1 & 1 & 1 & 1 & 1 & 1 & 1 & 1 & 1 & 1 & 0 & Gene transcription (Grace et al. 2004) \\
\hline (T)TGACY & 2 & 1 & 2 & 2 & 1 & 3 & 3 & 4 & 4 & 3 & 2 & 2 & 2 & Early plant defense/wounding (Eulgem et al. 1999; Nishiuchi et al. 2004) \\
\hline
\end{tabular}

Motifs: the 15 most overrepresented motifs (size-2-12 bp) among training set sequences $(n=13)$ were recognized by the MEME algorithm (Bailey et al. 2006). Known plant associated cis-elements in motif sequences were identified using PLACE signal scan (Higo et al. 1999). The numbers of cis-elements that are present in multiple copies are presented in the table

$O s G L P$ gene family 5 ' regulatory regions: 1,000 -bp sequences upstream of ATG initiation codons for $O s G L P 8-1$ to $8-12$ were extracted from the Nipponbare reference genome sequence, MSU Pseudomolecules, V.4

${ }^{\text {a }}$ The indica-specific OsGLP8-6 insertion sequence $(858 \mathrm{bp})$ was cloned and sequenced from cultivars IR64 and SHZ-2 and was included in the motif search training set 
Table 3 Nucleotide (SNP) and aa Substitutions Among $O s G L P$ Alleles from Four Rice Cultivars
All reported nucleotide and amino acid substitutions are in reference to allele sequences from the temperate japonica cultivar, LTH

a 3 -bp deletion/1 amino acid deletion

b 3-bp insertion/ 1 amino acid insertion

${ }^{\mathrm{c}} 1$-bp deletion / frameshift mutation/premature termination codon

${ }^{\mathrm{d}}$ Nucleotide substitution / premature termination codon

\begin{tabular}{|c|c|c|c|c|c|c|c|}
\hline \multirow{3}{*}{$O s G L P$} & \multirow[t]{3}{*}{ MSU locus ID } & \multicolumn{6}{|c|}{ Rice cultivars } \\
\hline & & \multicolumn{2}{|c|}{ Azucena } & \multicolumn{2}{|l|}{ IR64 } & \multicolumn{2}{|c|}{ SHZ-2 } \\
\hline & & SNPs & aa subs & SNPs & aa subs & SNPs & aa subs \\
\hline $8-1$ & LOC_Os08g08920 & 3 & 1 & 4 & 2 & $1^{\mathrm{c}}$ & - \\
\hline $8-2$ & LOC_Os08g08960 & 1 & 0 & 3 & $1^{\mathrm{a}}$ & 2 & 0 \\
\hline $8-3$ & LOC_Os08g08970 & 0 & 0 & 5 & 1 & 4 & 2 \\
\hline $8-4$ & LOC_Os08g08980 & 1 & 1 & 2 & 1 & 5 & 1 \\
\hline $8-5$ & LOC_Os08g08990 & 1 & 0 & 7 & $1^{\mathrm{b}}$ & 3 & 0 \\
\hline $8-6$ & LOC_Os08g09000 & 0 & 0 & 2 & 0 & 0 & 0 \\
\hline $8-7$ & LOC_Os08g09010 & 0 & 0 & 3 & 2 & 2 & 1 \\
\hline $8-8$ & LOC_Os08g09020 & 0 & 0 & 11 & 5 & 3 & 2 \\
\hline $8-9$ & LOC_Os08g09040 & 0 & 0 & 2 & 1 & 0 & 0 \\
\hline $8-10$ & LOC_Os08g09060 & 2 & 0 & 4 & 2 & 0 & 0 \\
\hline $8-11$ & LOC_Os08g09080 & 0 & 0 & 0 & 0 & $18^{\mathrm{d}}$ & - \\
\hline $8-12$ & LOC_Os08g13440 & 2 & 2 & 5 & 1 & 4 & 1 \\
\hline
\end{tabular}

not detected in any of the three leaf positions tested in any cultivar. The two resistant cultivars showed constitutive expression of $O s G L P 8-3$ in all leaf positions, while the susceptible variety showed no expression. Conversely, OsGLP8-9 was expressed only in Azucena and not in IR64 and SHZ-2. Some OsGLP family members exhibited developmental regulation of gene expression among leaf positions.

Table 4 Developmental Expression of $O s G L P$ Genes in Leaves of 21-Day-Old Rice Plants

\begin{tabular}{|c|c|c|c|c|c|c|c|c|c|}
\hline \multirow[t]{3}{*}{ Gene } & \multicolumn{9}{|c|}{ Leaf position ${ }^{a}$} \\
\hline & \multicolumn{3}{|c|}{ Azucena (-QTL) } & \multicolumn{3}{|c|}{ IR64 (+QTL) } & \multicolumn{3}{|c|}{ SHZ-2 (+QTL) } \\
\hline & 1 & 2 & 3 & 1 & 2 & 3 & 1 & 2 & 3 \\
\hline OsGLP8-1 & - & - & - & - & - & - & - & - & - \\
\hline$O s G L P 8-2$ & - & - & - & - & - & - & - & - & - \\
\hline OsGLP8-3 & - & - & - & + & + & + & + & + & + \\
\hline$O s G L P 8-4$ & + & + & - & + & + & + & + & - & + \\
\hline OsGLP8-5 & + & + & + & + & + & + & + & + & + \\
\hline OsGLP8-6 & - & + & - & - & - & + & + & - & + \\
\hline OsGLP8-7 & + & + & + & + & + & + & + & + & + \\
\hline OsGLP8-8 & - & + & - & - & - & - & - & - & + \\
\hline OsGLP8-9 & + & + & - & - & - & - & - & - & - \\
\hline OsGLP8-10 & - & - & - & - & - & - & - & - & - \\
\hline OsGLP8-11 & + & + & + & + & + & + & - & - & - \\
\hline OsGLP8-12 & + & + & + & + & + & + & + & + & + \\
\hline
\end{tabular}

Developmental expression: $(+)$ indicates presence of band in RT-PCR after 35 cycles

${ }^{\text {a }}$ Three leaf positions include youngest emerging leaf (1), second youngest, expanded leaf (2), and third youngest, mature leaf (3)
OsGLP genes are transiently induced in response to $M$. oryzae and wounding

To determine if differential expression of $O S G L P$ family members correlates with disease phenotypes, gene expression was tested by RT-PCR on a time course $(0,12,24$, and 48 hpi) after M. oryzae infection (Fig. 3). As observed in developmental studies, transcripts of OsGLP8-1 and 8-2 were not detected in any cultivar under any treatment or time point. Transcripts of GER3 subfamily members, OsGLP8-3 and OsGLP8-4, were observed in untreated tissue in SHZ-2 and IR64, respectively, but not in the other cultivars (Fig. 3). OsGLP8-12 was more highly expressed in untreated tissue of Azucena and LTH compared to IR64 and SHZ-2. Transient induction of OsGLP8-12 was observed at 12 and $24 \mathrm{hpi}$ in three of four cultivars.

GER4 subfamily genes were clearly induced in response to M. oryzae infection in all cultivars (Fig. 3). OsGLP8-5 and 8-7 were constitutively expressed and transiently induced by $M$. oryzae infection in all cultivars. Low baseline levels of $0 s G L P 8-8,8-9$, and 8-10 were present at $0 \mathrm{~h}$, and all were transiently induced at 12 and $24 \mathrm{hpi}$. Basal transcript levels among cultivars differed for OsGLP8-11, but elevated expression was observed at 12 and $24 \mathrm{hpi}$. As predicted, transcripts of $O s G L P 8-11$ were not observed from SHZ-2 likely because of the nonsense mutation in the gene sequence. SHZ-2 plants had a higher constitutive level of OsGLP8-6 transcripts compared to other cultivars (Figs. 3 and 4). This gene was induced at 12 and $24 \mathrm{~h}$ in all lines, but the relative induction levels were greater in resistant compared to susceptible cultivars.

Overall, OsGLP expression between resistant and susceptible alleles differs at two time points: $0 \mathrm{~h}$ and $48 \mathrm{hpi}$ (Figs. 3 and 4). The resistant cultivars constitutively 
Fig. $3 O s G L P$ genes are induced by rice blast infection. Gene expression patterns of $O s G L P$ genes after challenge with $M$. oryzae. Three-week-old plants of two susceptible, Azucena $(A)$ and LTH $(L)$, and two resistant, IR64 $(I)$ and SHZ-2 $(S)$, rice cultivars were challenged with the virulent $M$. oryzae isolate P06-6 and sampled at $0,12,24$, and 48 hpi. OsGLP transcript levels were assayed with gene-specific primers by RT-PCR. The average band intensities of three biological replications $(n=3)$ were normalized to the highest intensity per gene and are shown in the heat maps. OsGLP genes in both GER3 and GER4 subfamilies were induced by $M$. oryzae infection. Transcripts of $O s G L P 8-11$ were not observed in the most resistant cultivar, SHZ-2.

express different combinations of OsGLP genes compared to the susceptible. For the GER3 subfamily, the indica varieties utilize $O s G L P 8-3$ and $O s G L P 8-4$, while the japonicas express $O s G L P 8-12$. The most resistant cultivar, SHZ-2, is the only one to constitutively express OsGLP8-6 and also has the fewest number of genes induced at 48 hpi.

Most of the $O s G L P$ genes were also induced by the mock spray treatment (ESM Fig. 2), although to lower levels than after $M$. oryzae inoculation. To test whether induction of OsGLP genes by the mock treatment is consistent with response to wounding, a subset of OsGLP genes was tested by RT-PCR in leaf tissue after pressurized water spray and mechanical wounding (Fig. 5). Of the five genes tested, all were induced by both treatments, except for OsGLP8-11 which was not observed from SHZ-2. Overall, these results show that all OsGLP genes are induced by biotic and abiotic stimuli and that the timing and levels of induction vary among the different family members.

\section{OsGLP proteins are localized in the plant cell wall}

Fusion proteins of OsGLP8-6 and OsGLP8-12 with $\mathrm{C}^{\prime}$ terminal green fluorescent proteins (GFP) were visualized by transient expression in the heterologous plant, $N$. benthamiana (Fig. 6a). OsGLP-GFP proteins were coexpressed with a golgi-specific marker, G-rk CD3-967 (Nelson et al. 2007), to compare subcellular localizations. Both OsGLP-GFP fusion proteins showed similar punctate patterns to each other. In some cases, they associated with subcellular organelles at cell perimeters and co-localized with golgi-specific markers (Fig. 6a, merged images). The punctate, fluorescent patterns observed for OsGLP fusion proteins, however, were usually not located with the golgi markers and when viewed in multiple planes appeared to be associated with the cell surfaces (Fig. 6a, green channel and merged images).

Denatured monomer subunits of OsGLP fusion proteins were observed by immunoblot at the expected size of $55 \mathrm{kDa}$ (OsGLP predicted size, $24 \mathrm{kDa}$ ) compared to the control GFP protein of $26 \mathrm{kDa}$ (Fig. 6b). Two bands were observed for OsGLP8-12+C'GFP proteins, suggesting multiple isoforms of this protein.

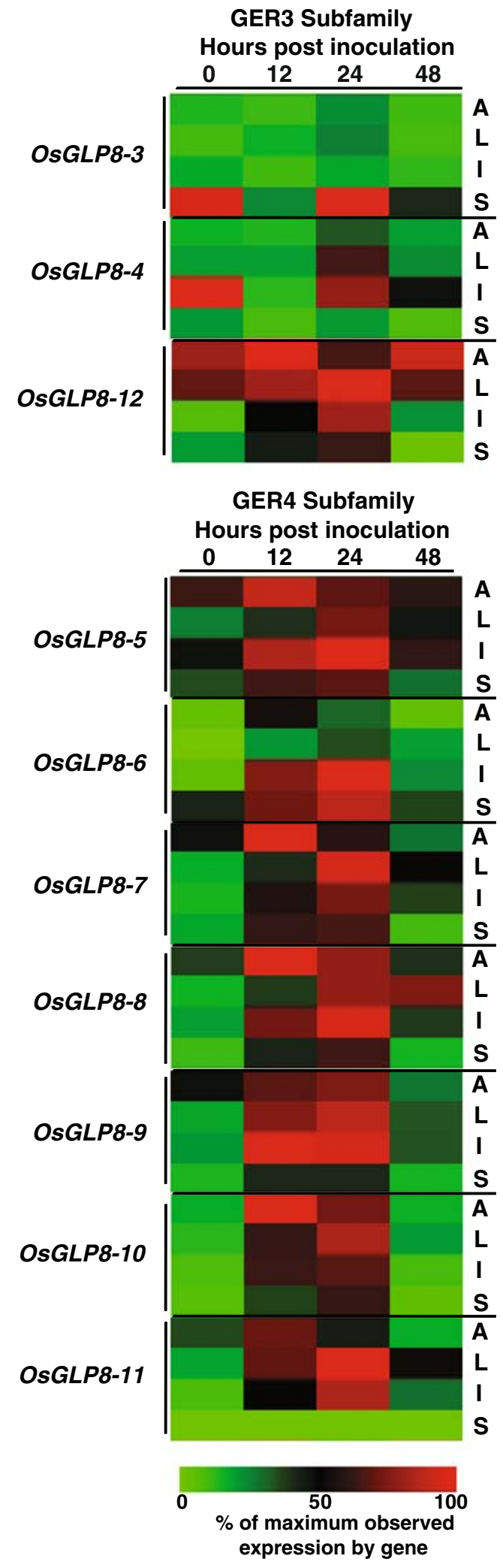



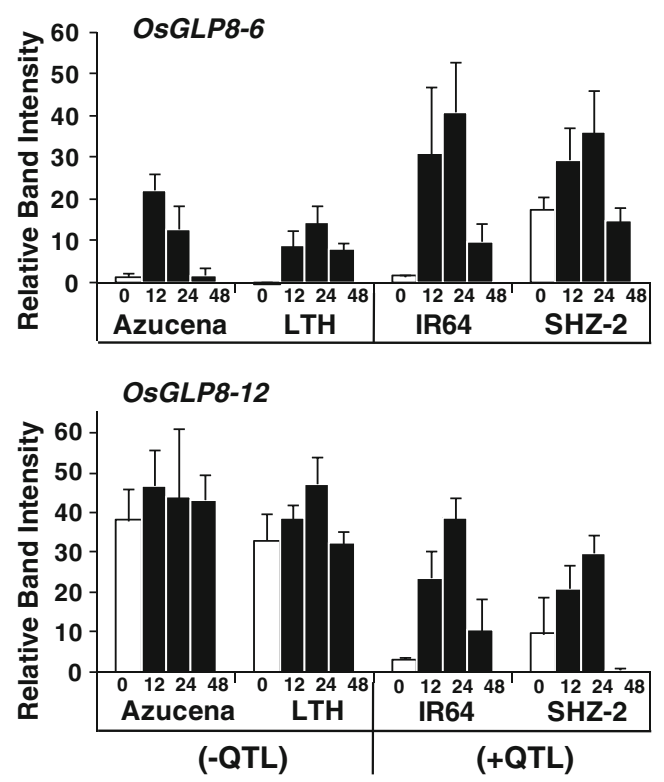

Fig. 4 Differential expression between resistant and susceptible cultivars was observed for two $O S G L P$ genes. a $O s G L P 8-6$ is upregulated in resistant cultivars. SHZ-2, a highly resistant cultivar, showed higher constitutive expression ( $0 \mathrm{hpi}$, white bars) than the other cultivars. Both resistant (+QTL) cultivars had higher relative induction by $M$. oryzae infection (black bars) compared to susceptible (-QTL). b OsGLP8-12 is upregulated in susceptible cultivars. There is high constitutive expression of OsGLP8-12 in Azucena and LTH (-QTL) compared to IR64 and SHZ-2 (+QTL). The resistant cultivars show peak induction of this gene at $24 \mathrm{hpi}$ with $M$. oryzae. OsGLP transcript levels were assayed with gene-specific primers by RT-PCR, and the average band intensities of three biological replications $(n=3)$ with standard errors are shown.

Resistant varieties produce $\mathrm{H}_{2} \mathrm{O}_{2}$ in early responses to rice blast infection

$\mathrm{H}_{2} \mathrm{O}_{2}$ was visualized in leaf tissue after rice blast inoculation by oxidation of 3,3'-diaminobenzidine (DAB; Fig. 7a). The SHZ-2 leaves showed a few DAB-stained spots as early as 12 hpi with $M$. oryzae, while the LTH leaves did not. Leaves of SHZ-2 also showed a higher number of DAB-stained spots at 24 and 48 hpi compared to LTH. Mock-inoculated plants sprayed with gelatin solution displayed fewer and fainter spots than the $M$. oryzaeinoculated leaves, suggesting that infection by $M$. oryzae induces $\mathrm{H}_{2} \mathrm{O}_{2}$ production.

To test for $\mathrm{H}_{2} \mathrm{O}_{2}$ production after abiotic stimulation, leaves of SHZ-2 were subjected to a gelatin spray treatment and mechanical wounding with a needle (ESM Fig. 3). $\mathrm{H}_{2} \mathrm{O}_{2}$ accumulated in faint spots after the water spray compared to the untreated, control leaf. There was also $\mathrm{H}_{2} \mathrm{O}_{2}$ accumulation in areas directly surrounding the needle wounds at $24 \mathrm{~h}$ post-treatment. These results show that rice leaves produce $\mathrm{H}_{2} \mathrm{O}_{2}$ after a variety of stimuli, including wounding and pathogen infection.

\section{Discussion}

We previously demonstrated that as more chr 8 OsGLP genes are silenced in a transgenic plant, the plants become more susceptible to $M$. oryzae and $R$. solani (Manosalva et al. 2009). This suggested that several of the 12 OsGLP genes within the QTL region contribute collectively to broad-spectrum disease resistance. Our objectives were to identify functional polymorphism(s) in $O S G L P$ alleles among rice blast resistant cultivars with the effective chr 8 QTL compared to susceptible cultivars without the chr 8 QTL. The resulting story is that of duplicated genes in two germin subfamilies with complex, cultivar-specific expression patterns. Biotic stress-inducible expression confirms that multiple $O s G L P$ s are pathogenesis-related proteins in rice as in other crop species (van Loon et al. 2006). The early temporal induction of OsGLPs and the location in the plant cell wall suggest that they play roles in early layers of plant defenses. The earlier expression of some GLP at higher levels in resistant versus susceptible cultivars corresponds with differences in early physiological defense responses such as $\mathrm{H}_{2} \mathrm{O}_{2}$ accumulation. We hypothesize that earlier and higher induction of certain OsGLP genes provides the main advantage to resistant cultivars as opposed to loss of function mutations in coding sequences of susceptible cultivars.

The 12 OsGLP genes within the QTL region (Fig. 1) represent two germin subfamilies, GER3 and GER4 (ESM Fig. 1). Members of these groups have been identified from several taxa (Davidson et al. 2009; Manosalva et al. 2009). Phylogenetic analyses indicate that both subfamilies were

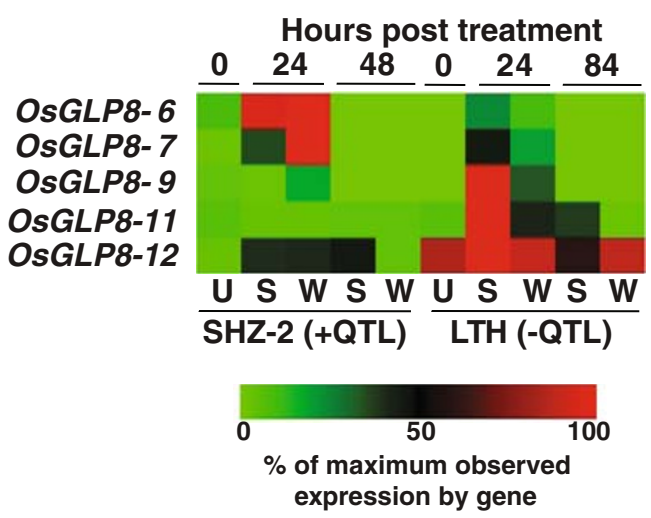

Fig. $5 O s G L P$ genes are differentially induced by mechanical wounding. Induction of selected $O S G L P$ genes after pressure spray $(S)$ and mechanical wounding $(W)$ compared to untreated controls $(U)$ in rice cultivars SHZ-2 and LTH. OSGLP transcript levels were assayed with gene-specific primers by RT-PCR, and average band intensities of three biological replications $(n=3)$ were normalized to the highest intensity per gene and are shown in the heat maps. Multiple $O S G L P$ genes were induced by both treatments in the two cultivars. Transcripts of $O s G L P 8-11$ were not observed in the most resistant cultivar, SHZ-2. 
Fig. 6 Subcellular localization of OsGLP8-6 and OsGLP8-12. a Each GLP protein was fused with a C'GFP tag and coexpressed with a golgi-specific marker protein with an mCherry tag (Nelson et al. 2007) in $N$. benthamiana via A. tumefaciens transformation. Epidermal peels were examined by fluorescence microscopy 4 days postinoculation with $A$. tumefaciens strains containing the constructs and images are shown at $\times 400$ magnification. Both GLP proteins show bright punctate patterns in green channel images. In some cases, GLP proteins colocalized with golgi marker proteins at cell margins (merged images). However, GLP proteins also showed distinct localizations in several planes of view across cell surfaces. b Fusion proteins were detected by immunoblot with anti-GFP antibodies. Both show expected sizes around $56 \mathrm{kDa}$ compared to GFP expressed in M. oryzae at $26 \mathrm{kDa}$ (Kankanala et al. 2007). OsGLP12+C'GFP showed two bands indicating multiple isoforms.
A.

OsGLP8-6+C'GFP

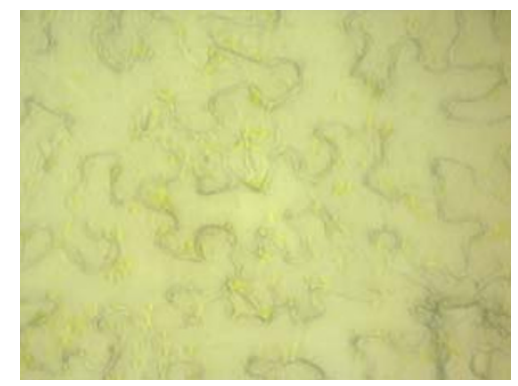

mCherry tag (red channel)
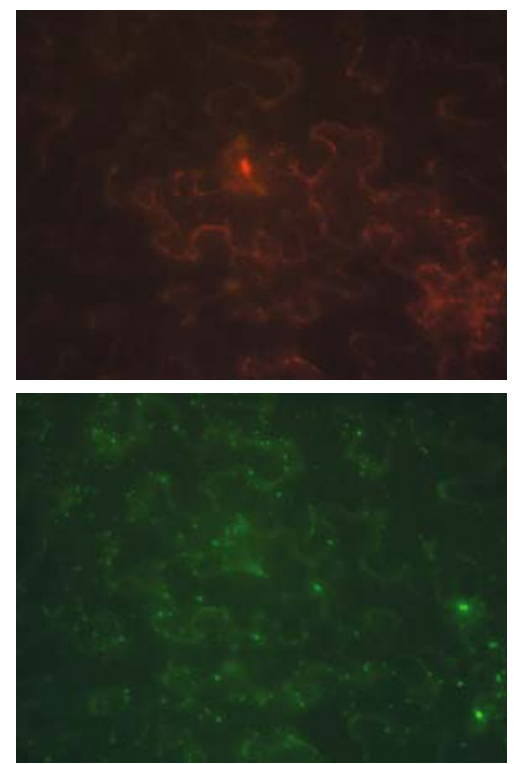

GFP tag (green channel)

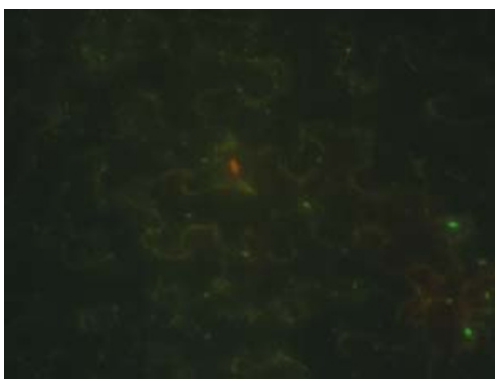

Merged Image
OsGLP8-12+C'GFP
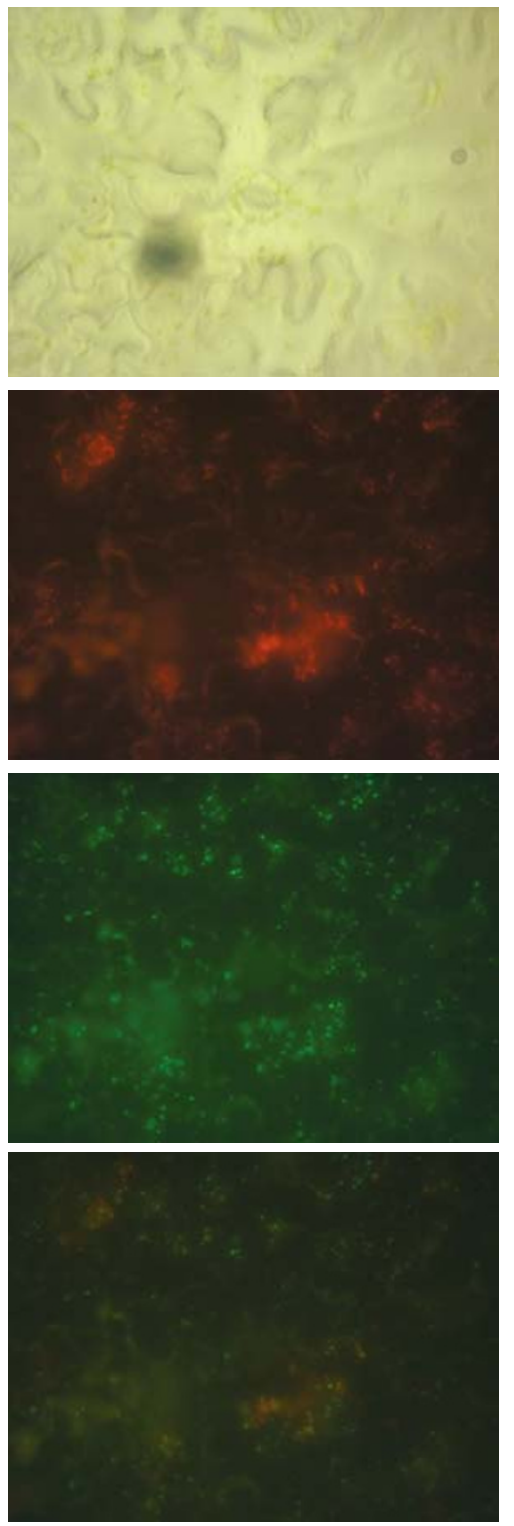

B.

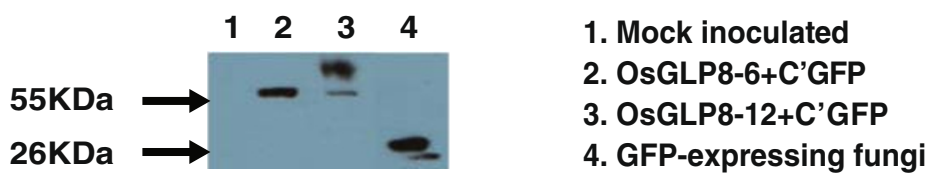

derived from a common ancestor and that gene duplication events occurred after separation of subfamily progenitors. The most recent duplication events occurred in the GER4 subfamily based on their close physical proximity to one another and similarities in gene and putative regulatory sequences (Fig. 1 and Table 2 and ESM Table 1). Allelic diversity occurs among $O S G L P$ genes within a single cultivar and among cultivars for a given OsGLP gene.
Therefore, the potential number of allelic combinations of $O s G L P$ s present among rice germplasm is very high.

We analyzed the variation in coding sequences among the alleles from the various cultivars. Most sequence variations correlated to subspecies pedigrees (japonica vs. indica), so we focused on mutations that putatively cause major effect functional changes. Of all putative amino acid (aa) substitutions and insertion/deletions identified (Table 3), none 


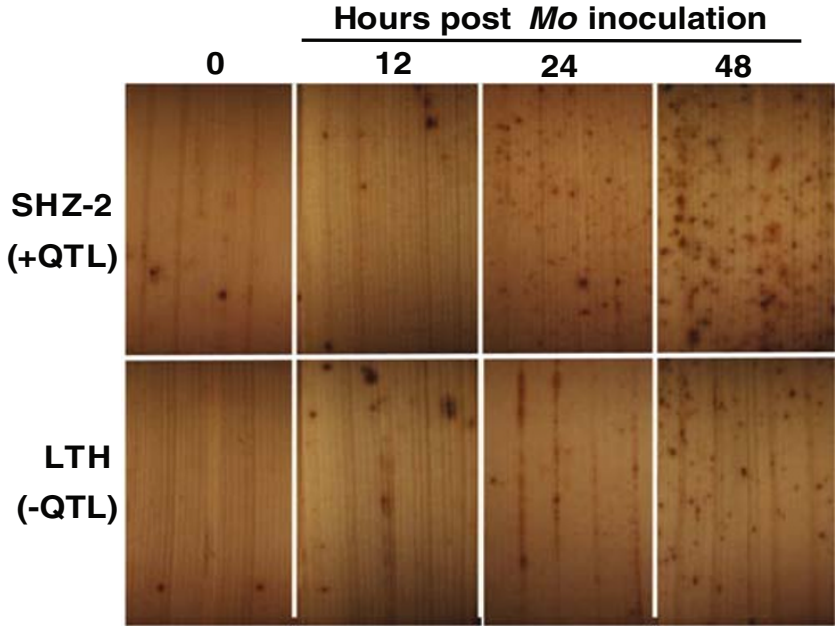

Fig. 7 Hydrogen peroxide $\left(\mathrm{H}_{2} \mathrm{O}_{2}\right)$ accumulation in leaf tissue of resistant and susceptible cultivars after inoculation with $M$. oryzae isolate P06-6. $\mathrm{H}_{2} \mathrm{O}_{2}$ was visualized using 3,3'-diaminobenzidine $(D A B)$; oxidation of DAB by $\mathrm{H}_{2} \mathrm{O}_{2}$ results in a dark brown color (dark spots on image). Twenty-one-day-old plants of highly resistant cultivar, SHZ-2, and susceptible cultivar, LTH, were spray-inoculated with $M$. oryzae, and leaves were collected at $0,12,24$, and 48 hpi. Leaves of SHZ-2 showed a higher number of DAB stained spots at 24 and 48 hpi compared to LTH.

were predicted in conserved functional domains such as active sites or N-terminal signal sequences. The most resistant cultivar, SHZ-2, had nonsense mutations in two genes (OsGLP8-1 and OsGLP8-11), consistent with lack of detectable transcripts. These mutations were not present in the moderately resistant cultivar, IR64, and gene silencing of OsGLP8-11 conferred some loss of resistance in the cultivar, Kitaake (Manosalva et al. 2009). It is unlikely that the loss of these genes provides an advantage to SHZ-2.

Analyses of putative $5^{\prime}$ regulatory regions suggested that GER4 subfamily members are most important for defense (Table 2), which is consistent with gene silencing studies (Manosalva et al. 2009). Gene expression profiling shows that many $O s G L P$ of the GER4 subfamily are induced after challenge with $M$. oryzae in all cultivars, although one GER3 (OsGLP8-12) is also induced (Figs. 2 and 3). In barley, $H v G E R 4$ transcripts accumulated rapidly, as early as $3 \mathrm{hpi}$, and were correlated with the formation of papilla, structures associated with defense responses (Wei et al. 1998; Zimmermann et al. 2006). We also observed induction of $O S G L P$ early in defense responses (12 hpi, Fig. 3), and this induction preceded the penetration of the cell wall by $M$. oryzae that occurs between 18 and $24 \mathrm{hpi}$ (Kankanala et al. 2007; Mosquera et al. 2009). The genes also showed sustained induction through $48 \mathrm{hpi}$, indicating that they remain present throughout pathogenesis.

Many $O s G L P$ were also induced by the mock spray treatment and mechanical wounding (Fig. 5 and ESM Fig. 2). The presence of multiple W-BOX transcription factor binding site motifs in $O s G L P$ promoters is consistent with the induction by wounding. WRKY transcription factors are rapidly induced by pathogen elicitors and in response to wounding and physically bind to W-BOX motifs (Eulgem et al. 1999; Nishiuchi et al. 2004). The GER4 subfamily member $5^{\prime}$ regulatory regions contained the most W-BOX motifs, consistent with their induction by wounding and $M$. oryzae. Some GER4 genes were also shown to be induced by inoculation with the bacterial blight pathogen Xanthomonas oryzae pv. oryzae in genome wide expression profiling experiments (Nobuta et al. 2007; Davidson et al. 2009).

Two obvious differences were observed between $O s G L P$ expression patterns in resistant and susceptible plants. The first includes constitutive expression and transient induction of OsGLP8-6, a GER4 subfamily member (Fig. 4). The coding region of this gene was highly conserved across all four cultivars, suggesting that it may be functionally important (Table 3). The promoter insertion sequences identified in the two indica cultivars likely influence the differential expression patterns, especially with the addition of two W-BOX motifs to the existing three copies. WRKY proteins bind cooperatively to clusters of W-BOX motifs (Eulgem 2006). Thus, we hypothesize that enhanced expression of OsGLP8-6 in resistant cultivars is conferred through this alternative promoter. The second difference was observed in expression of $O s G L P 8-12$ which was more highly expressed in susceptible versus resistant cultivars (Figs. 3 and 4). OsGLP8-12 is a member of the GER3 subfamily and was not detected as important for disease resistance in rice or barley (Zimmermann et al. 2006; Manosalva et al. 2009). In fact, transient silencing of $H v G E R 3$ resulted in enhanced resistance to $B$. graminis (Zimmermann et al. 2006). Scrutiny of genome-wide rice small RNA libraries suggests that there is opposite regulation of GER3 and GER4 genes (Davidson et al. 2009). Alternatively, there could be transcriptional suppression of GER4 genes through feedback inhibition. A reasonable hypothesis is that OsGLP8-12 expression suppresses GER4 family members, which results in a constitutive disadvantage for the susceptible cultivars.

OsGLP genes also show developmental regulation in leaves (Table 4) and were detected in cDNA libraries from panicles, shoots, seeds, and roots (Table 1; Membre and Bernier 1998). The presence of developmentally related ciselements in OsGLP promoters is consistent with these observations (Table 2; Yanagisawa and Schmidt 1999; Rogers et al. 2001). The detection of GLPs of rice and other species in plant cell walls is also consistent with their roles as mediators of cell wall expansion (Membre et al. 2000) and a role in cell wall reinforcement after stress induction (Vallelian-Bindschedler et al. 1998).

Highly related orthologs of OSGLP have SOD activity that converts unstable and transient superoxide anions 
into $\mathrm{H}_{2} \mathrm{O}$ (Woo et al. 2000; Christensen et al. 2004; Godfrey et al. 2007). Superoxide is produced quickly in the apoplast following recognition of pathogen elicitors by NAPDH oxidases and peroxidases (Wojtaszek 1997). In addition, M. oryzae requires fungal-produced superoxide for successful penetration of plant cuticles (Egan et al. 2007). If rice $O S G L P$ also exhibit SOD activity in the cell wall as predicted from studies of orthologous proteins (Wei et al. 1998; Christensen et al. 2004; Godfrey et al. 2007), then they likely produce some or all of the $\mathrm{H}_{2} \mathrm{O}_{2}$ produced after stress stimuli which may impede fungal penetration. Indeed, we observed $\mathrm{H}_{2} \mathrm{O}_{2}$ accumulation after the same stimuli that induced $O s G L P$ genes (Figs. 3 and 6). The earlier accumulation of $\mathrm{H}_{2} \mathrm{O}_{2}$ after infection with $M$. oryzae in the resistant cultivar, SHZ-2, compared to the susceptible, LTH, indicates that timing of the defense response is key to the resistance strategy. This is supported by observations of collective $O s G L P$ expression in which SHZ-2 has the earliest transient gene induction after $M$. oryzae infection and the fewest genes induced at $48 \mathrm{hpi}$.

The evidence presented here, as well as that from other monocot and dicot species, provide insights into the contributions of GLP to disease resistance QTL and the complexity of the gene family member regulation. Our data show that OSGLP genes, particularly GER4 subfamily members, are induced by pathogens in all cultivars but that temporal expression patterns differed. Corresponding with differential $O S G L P$ expression among cultivars were variations in the $5^{\prime}$ regulatory regions and differential accumulation of $\mathrm{H}_{2} \mathrm{O}_{2}$ after pathogen infection. Taken together, we conclude that $O s G L P$ play roles in early defense responses and that early induction may be beneficial for protecting plants at or near fungal penetration sites. With respect to breeding strategies, $O S G L P$ s may be effectively utilized by selecting for the resistant donor GER4 gene cluster including regulatory regions. This identification of genes contributing to broad-spectrum disease resistance facilitates the introgression of donor alleles into adapted cultivars.

\section{Materials and methods}

Plant materials

Rice cultivars, Azucena (ssp. japonica), Lijiangxin-tuanheigu (LTH; ssp. japonica), IR64 (ssp. indica), and Sanhuangzhan 2 (SHZ-2; ssp. indica) were grown in a Bacto soilless media (Michigan Peat Co., Houston, TX) for 21 days and fertilized once a week with $5 \mathrm{~g} / \mathrm{L} \mathrm{NH}_{4} \mathrm{SO}_{4}$. Plants were grown with a photoperiod of 16 -h light $/ 8$ $\mathrm{h}$ dark in a growth chamber and alternating day/night temperatures of $28 / 26^{\circ} \mathrm{C}$ and $80 \% \mathrm{RH}$.
Cloning of $O s G L P$ alleles

The 12 OsGLP were PCR-amplified from genomic DNA of four cultivars (Azucena, LTH, IR64, and SHZ-2) and cloned into pGEM-T (Promega, Madison, WI) using primers and annealing temperature described in Manosalva et al. (2009). Genes were sequenced from plasmids at a minimum of $4 \times$ coverage. Protein predictions were performed using FGENESH (http://linux1.softberry.com/berry.phtml?topic= fgenes_plus\&group=programs\&subgroup $=\mathrm{gfs}$ ).

$5^{\prime}$ regulatory region motif analysis

Putative regulatory sequences that are $5^{\prime}$ to $O s G L P$ genes (1 peroxidases upstream from initiation codon) for the 12 gene family members were extracted from the reference japonica genome sequence (cv. Nipponbare; MSU Pseudomolecules, Build 4.0; Yuan et al. 2005) using a custom perl script. Similar regions in the reference indica genome sequence (cv. 93-11, Zhao et al. 2004) were extracted through blastn searches. The putative $5^{\prime}$ regulatory sequences for the 12 OsGLP genes and the OsGLP8-6 insertion sequence ( $858 \mathrm{bp}$, only present in the indica sequence) were analyzed as a training set $(N=13)$ for statistically overrepresented sequence motifs using the MEME algorithm (Bailey et al. 2006; ESM Table 1; motif size $=2$ $11 \mathrm{bp}, 15$ motifs, +-- strand, $p<0.001)$. The 15 most significant motif sequences were searched against the PLACE database of 496 known plant-associated ciselements using PLACE Signal Scan (Higo et al. 1999). cis-elements within motif sequences were counted for all putative regulatory sequences in the training set, and those with multiple copies were summarized (Table 2).

Because sequence differences were observed in the $5^{\prime}$ regulatory regions of OSGLP8-6 between the two reference genomes (japonica vs. indica), the regions were amplified from each of the four cultivars with primers designed 639 and 85 bp upstream from start codon (Fig. 4b, c; GLP6proF2, 5' CGTTCAATTTTCTAAGCCAGATTGTG 3' and oxo6ProR 5' CTTCCCATCAGAGAAAGATAGCAG $\left.3^{\prime}\right)$. The sequences were cloned into pGEM-T (Promega) and sequenced at $4 \times$ coverage.

Fungal growth conditions, inoculation/wounding methods

Plants were inoculated with Philippine $M$. oryzae isolate PO6-6 that exhibits broad virulence to rice genotypes and is routinely used for the evaluation of quantitative resistance to blast (Wang et al. 1994; Liu et al. 2004; Manosalva et al. 2009). Cultures were grown on oatmeal agar media under constant light at $26^{\circ} \mathrm{C}$ for 21 days. Plants were sprayinoculated with $5 \times 10^{5}$ spores per milliliter suspended in a gelatin solution at 20 psi using an artist's air brush (Valent 
et al. 1986). Mock inoculation consisted of a spray with gelatin solution.

For the wounding experiments, LTH and SHZ-2 were grown for 21 days. The two youngest fully expanded leaves were pierced with a needle at $1-\mathrm{cm}$ intervals from the tip downward for $8 \mathrm{~cm}$.

\section{Plant harvest, RNA isolation and RT-PCR}

To monitor developmental expression of the $O s G L P$ genes, tissue was harvested from the three most fully expanded leaves from six 21-day-old plants per cultivar; six leaves from each position were pooled per cultivar. For expression experiments after $M$. oryzae inoculation, tissue was harvested by combining the three most fully expanded leaves pooled from two plants per cultivar. In the wounding experiments, the two youngest, wounded leaves were pooled from two plants per cultivar.

Samples were ground in liquid nitrogen, and total RNA was isolated using Trizol (Invitrogen, Carlsbad, CA). RNA was treated with one unit of DNase (Promega) per micrograms total RNA. Single-strand cDNA was synthesized from $2 \mu \mathrm{g}$ of total RNA using Superscript III reverse transcriptase (RT; Invitrogen) and 50 pmol $\operatorname{oligodT}_{(20)}$ primer. Gene-specific primers (10 pmol of each primer) were used in RT-PCR for 35 cycles at the appropriate annealing temperature (Manosalva et al. 2009). Total RNA was measured with a spectrophotometer, and $18 s$ rRNA or EF1-alpha were used as reference genes (Kim et al. 2003) to ensure equal RNA loading among samples.

Expression profiling by RT-PCR was performed from leaf tissue of three independent plant inoculation/wounding experiments. PCR products were fractionated on $0.8 \%$ agarose gels $(w / v)$ and stained with ethidium bromide. Stained gels were visualized and digitally photographed using the Syngene Gene Genius Bioimaging System, and gel band intensities were quantified using Gene Tools Gel Analysis software (Syngene, Frederick, MD). Band intensity values were normalized by subtracting the signal of the negative control in a given gel. Intensities for each gene/ treatment/cultivar combination were averaged over biological replicates $(n=3)$.

Construct design, transient protein expression, GFP microscopy, and immunodetection

OsGLP8-6 and OsGLP8-12 genes from cultivar SHZ-2 were amplified from $\mathrm{p}$-GEM-T plasmids using the primers (GLP6 prot F, 5' CACCATGGCTTCACCCTCTTCCCT 3', GLP6 prot R, 5' GTAGTGATTGTTCTCCCAGAAC 3', GLP12 prot F, 5'CACCATGGCCTCCTCTTCCT TATTTC 3', and GLP12 prot_R, 5' GTAGTTGTTCTCC CAGAACTGAG $3^{\prime}$ ) and were ligated into the entry vectors
(pENTR-D TOPO; Invitrogen). Positive entry clones were digested into two fragments using $M l u \mathrm{I}$ and were purified using sodium acetate precipitation. Plasmid fragments containing GLP genes were recombined into the GFP protein fusion plasmid, pEarleyGate103 (Earley et al. 2006) using LR Clonase (Invitrogen). After amplification in Escherichia coli, purified plasmids were transformed into Agrobacterium tumefaciens strain EHA105. An A. tumefaciens strain containing a plasmid encoding a golgi-specific protein marker with an mCherry tag called G-rk CD3-967 (Nelson et al. 2007).

Transformed A. tumefaciens strains containing one of three constructs (OsGLP6+C'GFP, OsGLP12+C'GFP, and Grk CD3-967) were grown in $20 \mathrm{ml}$ cultures according to (Sparkes et al. 2006). For co-expression experiments, two $A$. tumefaciens strains containing single constructs were mixed and infiltrated into leaves of Nicotiana benthamiana. Epidermal peels of transformed $N$. benthamiana leaves were imaged at $\times 400$ magnification with a compound epifluorescent microscope in bright field, green channel $(509 \mathrm{~nm})$ to observe GFP fluorescence and the red channel $(610 \mathrm{~nm})$ to observe mCherry fluorescence. Images were merged using Metamorph 6.1 imaging software (Downingtown, PA).

GFP fusion proteins were visualized by immunodetection. $N$. benthamiana leaves were ground in Laemmli buffer (BioRad, Hercules, CA) 4 days post-inoculation with A. tumefaciens containing the constructs (OsGLP6 $+\mathrm{C}^{\prime}$ GFP, OsGLP12+C'GFP). Crude protein extracts were boiled for $5 \mathrm{~min}$ and separated on 12\% SDS-PAGE gels. Proteins were transferred to a nylon membrane and were probed with anti-GFP and anti-rabbit antibodies. Antibodies were detected with West Pico Chemi-luminescent Substrate (Thermo Scientific, Waltham, MA).

Hydrogen peroxide $\left(\mathrm{H}_{2} \mathrm{O}_{2}\right)$ staining

$\mathrm{H}_{2} \mathrm{O}_{2}$ accumulation was visualized in leaf tissue using DAB as described (Thordal-Christensen et al. 1997). Twentyone-day-old plants of cultivars LTH and SHZ-2 were sprayinoculated with M. oryzae P06-6. Leaves were collected at three time points after inoculation and then stained with DAB. Leaves from SHZ-2 were subjected to a mock spray treatment or mechanical wounding with a needle, were harvested $24 \mathrm{~h}$ post-treatment, and then stained with DAB. Leaves were cleared of chlorophyll in 95\% ethanol and digital images were taken with a dissecting microscope.

Acknowledgments We thank Kevin Childs for writing perl script used in the study. Rebecca Davidson was supported by a Ford Foundation Diversity Fellowship and a USAID Linkage Project. Jacob Snelling was supported by USDA-CSREES-NRI-Rice-CAP grant 2004-35317-14867, and Patricia Manosalva and Myron Bruce were supported by USDA-CSREES-NRI grants 2003-01551 and 200635604-16628, respectively. 


\section{References}

Bailey TL, Williams N, Misleh C, Li WW. MEME: discovering and analyzing DNA and protein sequence motifs. Nucleic Acids Res. 2006;34:W369-373.

Ballini E, Morel JB, Droc G, Price A, Courtois B, Notteghem JL, et al. A genome-wide meta-analysis of rice blast resistance genes and quantitative trait loci provides new insights into partial and complete resistance. Mol Plant Microbe Interact. 2008;21:859-68.

Christensen A, Thordal-Christensen H, Zimmermann G, Gjetting T, Lyngkjaer M, Dudler R, et al. The germin-like protein GLP4 exhibits superoxide dismutase activity and is an important component of quantitative resistance in wheat and barley. Mol Plant Microbe Interact. 2004;17:109-17.

Davidson RM, Reeves P, Manosalva PM, Leach JE. Germins: a diverse protein family important for crop improvement. Plant Sci. 2009; 177:499-510.

Dunwell JM, Gibbings JG, Mahmood T, Naqvi SMS. Germin and germin-like proteins: evolution, structure, and function. Crit Rev Plant Sci. 2008;27:342-75.

Earley KW, Haag JR, Pontes O, Opper K, Juehne T, Song K, et al. Gateway-compatible vectors for plant functional genomics and proteomics. Plant J. 2006;45:616-29.

Egan MJ, Wang ZY, Jones MA, Smirnoff N, Talbot NJ. Generation of reactive oxygen species by fungal NADPH oxidases is required for rice blast disease. Proc Natl Acad Sci U S A. 2007;104:11772-7.

Eulgem T. Dissecting the WRKY web of plant defense regulators. PLoS Pathog. 2006;2:e126.

Eulgem T, Rushton PJ, Schmelzer E, Hahlbrock K, Somssich IE. Early nuclear events in plant defence signalling: rapid gene activation by WRKY transcription factors. EMBO J. 1999;18:4689-99.

Federico M, Iniguez-Luy F, Skadsen R, Kaeppler H. Spatial and temporal divergence of expression in duplicated barley germinlike protein-encoding genes. Genetics. 2006;174:179-90.

Gechev TS, Van Breusegem F, Stone JM, Denev I, Laloi C. Reactive oxygen species as signals that modulate plant stress responses and programmed cell death. Bioessays. 2006;28:1091-101.

Godfrey D, Able A, Dry I. Induction of a grapevine germin-like protein $(V v G L P 3)$ gene is closely linked to the site of Erysiphe necator infection: a possible role in defense? Mol Plant Microbe Interact. 2007;20:1112-25.

Grace ML, Chandrasekharan MB, Hall TC, Crowe AJ. Sequence and spacing of TATA box elements are critical for accurate initiation from the beta-phaseolin promoter. J Biol Chem. 2004;279:8102-10.

Grant JJ, Yun BW, Loake GJ. Oxidative burst and cognate redox signalling reported by luciferase imaging: identification of a signal network that functions independently of ethylene, SA and Me-JA but is dependent on MAPKK activity. Plant J. 2000;24:569-82.

Higo K, Ugawa Y, Iwamoto M, Korenaga T. Plant cis-acting regulatory DNA elements (PLACE) database: 1999. Nucleic Acids Res. 1999;27:297-300.

Kankanala P, Czymmek K, Valent B. Roles for rice membrane dynamics and plasmodesmata during biotrophic invasion by the blast fungus. Plant Cell. 2007;19:706-24.

Kim BR, Nam HY, Kim SU, Kim SI, Chang YJ. Normalization of reverse transcription quantitative-PCR with housekeeping genes in rice. Biotechnol Lett. 2003;25:1869-72.

Leung H. Stressed genomics-bringing relief to rice fields. Curr Opin Plant Biol. 2008;11:201-8

Liu B, Zhang S, Zhu X, Yang Q, Wu S, Mei M, et al. Candidate defense genes as predictors of quantitative blast resistance in rice. Mol Plant Microbe Interact. 2004;17:1146-52.

Mahmood T, Hyder MZ, Naqvi SM. Cloning and sequence analysis of germin-like protein gene 2 promoter from Oryza sativa L. ssp. indica. DNA Seq. 2007;18:26-32.
Manosalva PM, Davidson RM, Liu B, Zhu X, Hulbert SH, Leung H, et al. A germin-like protein gene family functions as a complex quantitative trait locus conferring broad-spectrum disease resistance in rice. Plant Physiol. 2009;149:286-96.

Membre N, Bernier F. The rice genome expresses at least six different genes for oxalate oxidase/germin-like proteins. Plant Physiol. 1998; $116: 868$

Membre N, Bernier F, Staiger D, Berna A. Arabidopsis thaliana germin-like proteins: common and specific features point to a variety of functions. Planta. 2000;211:345-54.

Mosquera $\mathrm{G}$, Giraldo $\mathrm{MC}$, Khang $\mathrm{CH}$, Coughlan S, Valent B. Interaction transcriptome analysis identifies Magnaporthe oryzae BAS1-4 as biotrophy-associated secreted proteins in rice blast disease. Plant Cell. 2009;21:1273-90.

Nelson BK, Cai X, Nebenfuhr A. A multicolored set of in vivo organelle markers for co-localization studies in Arabidopsis and other plants. Plant J. 2007;51:1126-36.

Nishiuchi T, Shinshi H, Suzuki K. Rapid and transient activation of transcription of the ERF3 gene by wounding in tobacco leaves: possible involvement of NtWRKYs and autorepression. J Biol Chem. 2004;279:55355-61.

Nobuta K, Venu RC, Lu C, Belo A, Vemaraju K, Kulkarni K, et al. An expression atlas of rice mRNAs and small RNAs. Nat Biotechnol. 2007;25:473-7.

Paran I, Zamir D. Quantitative traits in plants: beyond the QTL. Trends Genet. 2003;19:303-6.

Poland JA, Balint-Kurti PJ, Wisser RJ, Pratt RC, Nelson RJ. Shades of gray: the world of quantitative disease resistance. Trends Plant Sci. 2009;14:21-9.

Ramalingam J, Vera Cruz CM, Kukreja K, Chittoor JM, Wu JL, Lee $\mathrm{SW}$, et al. Candidate defense genes from rice, barley, and maize and their association with qualitative and quantitative resistance in rice. Mol Plant Microbe Interact. 2003;16:14-24.

Rogers HJ, Bate N, Combe J, Sullivan J, Sweetman J, Swan C, et al. Functional analysis of cis-regulatory elements within the promoter of the tobacco late pollen gene g10. Plant Mol Biol. 2001;45:577-85.

Schweizer P, Christoffel A, Dudler R. Transient expression of members of the germin-like gene family in epidermal cells of wheat confers disease resistance. Plant J. 1999;20:540-52.

Shetty NP, Jorgensen HJL, Jensen JD, Collinge DB, Shetty HS. Roles of reactive oxygen species in interactions between plants and pathogens. Eur J Plant Pathol. 2008;121:267-80.

Sparkes IA, Runions J, Kearns A, Hawes C. Rapid, transient expression of fluorescent fusion proteins in tobacco plants and generation of stably transformed plants. Nat Protoc. 2006;1:2019-25.

Thordal-Christensen H, Zhang Z, Wei Y, Collinge D. Subcellular localization of $\mathrm{H}_{2} \mathrm{O}_{2}$ in plants. $\mathrm{H}_{2} \mathrm{O}_{2}$ accumulation in papillae and hypersensitive response during the barley-powdery mildew interaction. Plant J. 1997;11:1187-94.

Valent B, Crawford M, Weaver C, Chumley F. Genetic studies of pathogenicity and fertility of Magnaporthe grisea. Iowa State J Res. 1986;60:569-94.

Vallelian-Bindschedler L, Mosinger E, Metraux JP, Schweizer P. Structure, expression and localization of a germin-like protein in barley (Hordeum vulgare L.) that is insolubilized in stressed leaves. Plant Mol Biol. 1998;37:297-308.

van Loon LC, Rep M, Pieterse CMJ. Significance of inducible defenserelated proteins in infected plants. Annu Rev Phytopathol. 2006;44:135-62.

Wang G, Mackill DJ, Bonman JM, McCouch SR, Nelson RJ. RFLP mapping of genes conferring complete and partial resistance to blast resistance in a durably resistant rice cultivar. Genetics. 1994;136:1421-34.

Wei Y, Zhang Z, Andersen C, Schmelzer E, Gregersen P, Collinge D, et al. An epidermis/papilla-specific oxalate oxidase-like protein 
in the defense response of barley attacked by the powdery mildew fungus. Plant Mol Biol. 1998;36:101-12.

Willocquet L, Elazegui FA, Castilla N, Fernandez L, Fischer KS, Peng $\mathrm{S}$, et al. Research priorities for rice pest management in tropical Asia: a simulation analysis of yield losses and management efficiencies. Phytopathology. 2004;94:672-82.

Wojtaszek P. Oxidative burst: an early plant response to pathogen infection. Biochem J. 1997;322:681-92.

Woo E, Dunwell J, Goodenough P, Marvier A, Pickersgill R. Germin is a manganese containing homohexamer with oxalate oxidase and superoxide dismutase activities. Nat Struct Biol. 2000;7:103640.

Wu JL, Sinha PK, Variar M, Zheng KL, Leach JE, Courtois B, et al. Association between molecular markers and blast resistance in an advanced backcross population of rice. Theor Appl Genet. 2004;108:1024-32.

Yanagisawa S, Schmidt RJ. Diversity and similarity among recognition sequences of Dof transcription factors. Plant J. 1999;17: 209-14.

Yuan Q, Ouyang S, Wang A, Zhu W, Maiti R, Lin H, et al. The institute for genomic research Osal rice genome annotation database. Plant Physiol. 2005;138:18-26.

Zhao W, Wang J, He X, Huang X, Jiao Y, Dai M, et al. BGI-RIS: an integrated information resource and comparative analysis workbench for rice genomics. Nucleic Acids Res. 2004;32:D377-82.

Zimmermann G, Baumlein H, Mock H, Himmelbach A, Schweizer P. The multigene family encoding germin-like proteins of barley. Regulation and function in basal host resistance. Plant Physiol. 2006;142:181-92. 